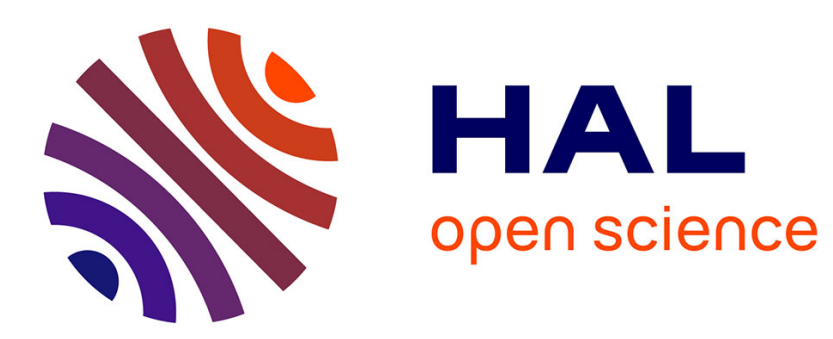

\title{
On some Riemannian aspects of two and three-body controlled problems
}

\author{
Jean-Baptiste Caillau, Bilel Daoud, Joseph Gergaud
}

\section{To cite this version:}

Jean-Baptiste Caillau, Bilel Daoud, Joseph Gergaud. On some Riemannian aspects of two and threebody controlled problems. 2009. hal-00432635v1

\section{HAL Id: hal-00432635 \\ https://hal.science/hal-00432635v1}

Preprint submitted on 23 Nov 2009 (v1), last revised 1 Jun 2010 (v2)

HAL is a multi-disciplinary open access archive for the deposit and dissemination of scientific research documents, whether they are published or not. The documents may come from teaching and research institutions in France or abroad, or from public or private research centers.
L'archive ouverte pluridisciplinaire HAL, est destinée au dépôt et à la diffusion de documents scientifiques de niveau recherche, publiés ou non, émanant des établissements d'enseignement et de recherche français ou étrangers, des laboratoires publics ou privés. 


\title{
On some Riemannian aspects of two and three-body controlled problems*
}

\author{
J.-B. Caillau ${ }^{1}$, B. Daoud ${ }^{1}$, and J. Gergaud ${ }^{2}$ \\ 1 Math. Institute, Bourgogne Univ. \& CNRS \\ \{jean-baptiste.caillau, bilel.daoud\}@u-bourgogne.fr \\ 2 ENSEEIHT-IRIT, Toulouse Univ. \& CNRS gergaud@enseeiht.fr \\ Dedicated to Joseph Noailles
}

\begin{abstract}
Summary. The flow of the Kepler problem (motion of two mutually attracting bodies) is known to be geodesic after the work of Moser [20], extended by Belbruno and Osipov $[2,21]$ : Trajectories are reparameterizations of minimum length curves for some Riemannian metric. This is not true anymore in the case of the three-body problem, and there are topological obstructions as observed by McCord et al. [19]. The controlled formulations of these two problems are considered so as to model the motion of a spacecraft within the influence of one or two planets. The averaged flow of the (energy minimum) controlled Kepler problem with two controls is shown to remain geodesic. The same holds true in the case of only one control provided one allows singularities in the metric. Some numerical insight into the control of the circular restricted three-body problem is also be given.
\end{abstract}

Key words: Two and three-body problems, geodesic flow, optimal control, cut and conjugate loci

MSC classification. 49K15, 53C20, 70Q05

\section{Introduction}

The circular restricted three-body problem is defined as follows [25].

Two bodies describe circular orbits around their center of mass under the influence of their mutual gravitational attraction, and a third one (attracted by the previous two but not influencing their motion) evolves

\footnotetext{
* The first author is supported by Conseil Régional de Bourgogne (contract no. 2009-160E-160-CE-160T) and ANR GCM. The second author is supported by the French Ministry for Higher Education \& Research (grant no. 31716-2008).
} 
in the plane defined by the two rotating ones. The restricted problem is to describe the motion of this third body.

We investigate the optimal control of this problem. The two primaries are planets, typically Earth and Moon, the third body is a spacecraft. The control is the thrust of this spacecraft. A recent example of this problem is the SMART-1 mission [4, 22] of the European Space Agency in the Earth-Moon system. This case has three important features: (i) provided we neglect noncoplanar effects, the circular restricted model is germane to the problem as the eccentricity of the Moon orbit is about $5.49 e-2$; (ii) the mass $m_{2}$ of the second primary (the Moon) is much smaller than the mass of the first (the Earth), $m_{1}$, so that $\mu=m_{2} /\left(m_{1}+m_{2}\right) \simeq 1.21 e-2$ is a small parameter of the model; (iii) the thrust of the engine is very low since solar-electric propulsion is used (around $7 e-2$ Newtons for a 350 Kilogram vehicle), so the magnitude of the control is another small parameter.

In a rotating frame, the dynamics is normalized to the second order mechanical system [25]

$$
\ddot{q}+\nabla V_{\mu}(q)+2 i q=\varepsilon u, \quad|u|=\sqrt{u_{1}^{2}+u_{2}^{2}} \leq 1 .
$$

Coordinate $q \in \mathbf{C} \simeq \mathbf{R}^{2}$ is the position vector, while $u$ is the control (the normalized acceleration, here). In this moving frame, the circular restricted three-body potential is

$$
\begin{gathered}
V_{\mu}(q)=-\frac{q^{2}}{2}-\frac{1-\mu}{r_{1}}-\frac{\mu}{r_{2}}, \\
r_{1}^{2}=\left(q_{1}+\mu\right)^{2}+q_{2}^{2}, \quad r_{2}^{2}=\left(q_{1}-1+\mu\right)^{2}+q_{2}^{2} .
\end{gathered}
$$

Parameter $\mu$ is the ratio $m_{2} /\left(m_{1}+m_{2}\right)$ of the masses of the two primaries, while $\varepsilon$ is the bound on the acceleration. When $\mu$ vanishes, we have a controlled two-body (Kepler) problem. The uncontrolled equations of motion can also be written in Hamiltonian form using Jacobi first integral (total energy),

$$
J_{\mu}(q, \dot{q})=\frac{|\dot{q}|^{2}}{2}+V_{\mu}(q) .
$$

In complex notation, let $p=\dot{q}+i q$. Then

$$
J_{\mu}(q, p)=\frac{|p|^{2}}{2}+p_{2} q_{1}-p_{1} q_{2}-\frac{1-\mu}{r_{1}}-\frac{\mu}{r_{2}} .
$$

The controlled system with Hamiltonian drift is so

$$
\dot{q}=\frac{\partial J_{\mu}}{\partial p}, \quad \dot{p}=-\frac{\partial J_{\mu}}{\partial q}+\varepsilon u, \quad|u| \leq 1 .
$$

In the case of two bodies $(\mu=0)$ and no control $(\varepsilon=0)$, the equations of motion in a fixed frame are 


$$
\ddot{q}+\frac{q}{|q|^{3}}=0, \quad q \in \mathbf{R}^{2}-\{0\} .
$$

In Hamiltonian form,

$$
\dot{q}=\frac{\partial J_{0}}{\partial p}, \quad \dot{p}=-\frac{\partial J_{0}}{\partial q},
$$

with energy $J_{0}=|\dot{q}|^{2} / 2-1 /|q|=|p|^{2} / 2-1 /|q|$, as $p=\dot{q}$ in the fixed frame. It was proven in [20] that, for negative values of the energy, the Hamiltonian flow of the system is a reparameterization of the geodesic flow on the punctured two-sphere, $\hat{\mathbf{S}}^{2}=\mathbf{S}^{2}-\{N\}$ (North pole removed). We sketch the construction in dimension $n \geq 2$ where the result holds unchanged. (Take $q \in \mathbf{R}^{n}-\{0\}$ in (2).) One first identifies the tangent bundle of the punctured $n$-sphere with the set of vectors $\xi=\left(\xi_{0}, \ldots, \xi_{n}\right), \eta=\left(\eta_{0}, \ldots, \eta_{n}\right)$ of $\mathbf{R}^{n+1}$ such that

$$
|\xi|=1, \quad(\xi \mid \eta)=0 .
$$

The puncture is obtained by removing $\xi_{0}=1$. Then, the transformation from the tangent bundle to $\mathbf{R}^{2 n}$ is

$$
q_{i}=\left(1-\xi_{0}\right) \eta_{i}+\eta_{0} \xi_{i}, \quad p_{i}=-\frac{\xi_{i}}{1-\xi_{0}}, \quad i=1, \ldots, n .
$$

Provided time is changed according to

$$
\mathrm{d} t=|q| \mathrm{d} s,
$$

the Hamiltonian flow on $J_{0}=-1 / 2$ is mapped into the Hamiltonian flow on $\widetilde{J}_{0}=1 / 2 \subset T \hat{\mathbf{S}}^{n}$ where

$$
\widetilde{J}_{0}(\xi, \eta)=\frac{1}{2}|\xi|^{2}|\eta|^{2} .
$$

This level set is the unit or spherical tangent bundle of $\hat{\mathbf{S}}^{2}$ since $|\eta|=1$. There,

$$
\xi^{\prime}=\eta, \quad \eta^{\prime}=-\xi,
$$

so $\xi^{\prime \prime}+\xi=0$ and one actually gets geodesics parameterized by arc length. The Levi-Civita change in time (3) regularizes the collision and the dynamics is extended on the whole $n$-sphere. The result of [20] was further generalized to cover the case of zero or positive energy levels by [2] and [21].

Trajectories in optimal control are projections of Hamiltonian flows, in general with singularities described by Pontryagin maximization condition. Riemannian problems being the simplest instance of control problems, one may ask wether a given Hamiltonian flow is the reparameterization of some Riemannian flow as in the two-body case. This question is addressed in [19], noting the following fact. Given a flow on an odd $2 n-1$-dimensional manifold $M$, a necessary condition for the flow to be geodesic is that the manifold be the unit tangent bundle of some other manifold of dimension $n$. This puts topological restrictions on $M$. These conditions are expressed in terms of the 
homology of $M$ and applied to the (general) three-body problem. We state the results and recall some basic facts on homology [16].

On a topological space $X$, a singular p-simplex is a continuous map $\sigma_{p}$ : $\Delta_{p} \rightarrow X$. Here, $\Delta_{p}$ is the standard $p$-simplex, that is the set of $\left(t_{0}, \ldots, t_{p}\right) \in$ $\mathbf{R}^{p+1}$ such that

$$
t_{0}+\cdots+t_{p}=1, \quad t_{i} \geq 0, \quad i=0, \ldots, p .
$$

Let $v_{0}, \ldots, v_{p}$ be its vertices. The set $C_{p}(X)$ of $p$-chains is the free abelian group generated by singular $p$-simplices. The boundary operator $\partial_{p}: C_{p}(X) \rightarrow$ $C_{p-1}(X)$ is

$$
\partial_{p}\left(\sigma_{p}\right)=\sum_{i=0}^{p}(-1)^{p} \sigma_{p} \mid \Delta\left(v_{0}, \ldots, \hat{v}_{i}, \ldots, v_{p}\right)
$$

where the restriction is on the $(p-1)$-simplex $\Delta\left(v_{0}, \ldots, \hat{v}_{i}, \ldots, v_{p}\right)$ with vertex $v_{i}$ removed, implicitly identified with $\Delta_{p-1}$. Images of $(p+1)$-chains by $\partial_{p+1}$ are $p$-boundaries, and $p$-chains in the kernel of $\partial_{p}$ are $p$-cycles. As $\delta_{p} \delta_{p+1}=0$, boundaries are cycles while, conversely, one defines the $p$-th homology group $H_{p}(X)$ as the quotient

$$
H_{p}(X)=\operatorname{Ker} \partial_{p} / \operatorname{Im} \partial_{p+1} .
$$

The rank of the $\mathbf{Z}$-module $H_{p}(X)$ is $\beta_{p}$, the $p$-th Betti number, and the EulerPoincaré characteristic of $M$ is

$$
\chi(M)=\sum_{p=0}^{n} \beta_{p} .
$$

Proposition 1 ([19]). If $M$ is a non-compact connected orientable manifold of dimension $2 n-1$, a necessary condition for it to be the unit tangent bundle of some orientable $n$-manifold is $\beta_{n-1} \neq 0$.

Applying this condition to the three-body problem, one gets the following negative result.

Theorem 1 ([19]). The flow of the planar three-body problem on a negative level of energy is not geodesic.

In the case of controlled two and three-body problems, there is not much hope to retrieve Riemannian flows, unless one uses some approximation process. The paper is organized as follows. In section 2, we examine the case of two bodies and two controls. Using averaging on a relaxed problem, we show that the flow is Riemannian when the $\mathrm{L}^{2}$-norm of the control is minimized. Its properties are essentially captured by those of a metric on the two-sphere. The same holds true for the case of two bodies and one control (directed by velocity) provided one allows singularities in the metric. This is addressed in section 3. A preliminary discussion of the three-body and two-control case 
is made in section 4 . The problem is sub-Riemannian with drift. One can still define the exponential mapping associated with minimum time extremals and compute conjugate points to ensure, as in the Riemannian case, local optimality of trajectories.

\section{Two bodies, two controls}

We consider an $\mathrm{L}^{2}$-relaxation of the controlled two-body problem. The bound $\varepsilon$ on the control is dropped,

$$
\ddot{q}+\frac{q}{|q|^{3}}=u, \quad u \in \mathbf{R}^{2},
$$

while the final time, $t_{f}$, is a fixed parameter of the criterion:

$$
\int_{0}^{t_{f}}|u|^{2} \mathrm{~d} t \rightarrow \min
$$

In the sub-Riemannian case, $\mathrm{L}^{2}$-minimization parameterized by final time can be recast as a minimum time problem with a bound on the control. Both problems coincide, so $t_{f}$ and $\varepsilon$ play dual roles in this sense. The situation is more complicated here because of the Kepler drift in the motion. In order to identify a new small parameter of the problem and perform averaging, we notice that the negative energy level $J_{0}<0$ has a trivial fiber structure. This is apparent in suited geometric coordinates.

The set $X$ of oriented ellipses has moduli space the product manifold $\mathbf{R}_{+}^{*} \times \mathbf{S}^{2}$ : Each ellipse is defined by its semi-major axis $a>0$ (we exclude trivial orbits, $a=0$ ), and to any point on $\mathbf{S}^{2},(\theta, \varphi)$ in standard spherical coordinates, is uniquely associated an eccentricity, $e=\sin \varphi$, an argument of perigee (angle of the semi-major axis with a fixed reference axis), $\theta$, and an orientation. The orientation of the ellipse changes when the point goes from one hemisphere to the other. Collisions orbits correspond to the equator $\varphi=\pi / 2$ and are included in the model.

Remark 1. Ellipses associated with the poles or the equator have richer symmetries (automorphisms) than others. The moduli space is then said to be coarse. It remains finer that the moduli space of conformal ellipses where homothety and rotation moduli $(a, \theta)$ would be dropped.

Position on the orbit is defined by the polar angle in the plane or longitude, $l \in \mathbf{S}^{1}$. The state space is hence $\mathbf{S}^{1} \times X$, and we have a trivial fiber space whose fiber is the moduli space. To each uncontrolled trajectory on $J_{0}<0$ corresponds a unique point in the fiber, so the drift in (4) has the form

$$
F_{0}(l, x)=\omega(l, x) \frac{\partial}{\partial l}, \quad(l, x) \in \mathbf{S}^{1} \times X .
$$


Keeping the same notation, let then $l$ be the cumulated longitude, associated with the covering

$$
\mathbf{R} \ni l \mapsto e^{i l} \in \mathbf{S}^{1} .
$$

Choosing $l$ as new time, we recast the problem as a periodic sub-Riemannian problem on $X$,

$$
\begin{gathered}
\frac{\mathrm{d} x}{\mathrm{~d} l}=u_{1} F_{1}(l, x)+u_{2} F_{2}(l, x), \quad u \in \mathbf{R}^{2}, \\
\int_{0}^{l_{f}}|u|^{2} \frac{\mathrm{d} l}{\omega(l, x)} \rightarrow \min \quad \text { (fixed } l_{f} \text { ). }
\end{gathered}
$$

The two vector fields $F_{1}, F_{2}$ on $X$ are periodic in the parameter $l$. Introducing mean motion, $n=a^{-3 / 2}$, and true anomaly, $\tau=l-\theta$, one gets

$$
\begin{aligned}
F_{1}(l, x)= & \frac{P^{2}}{W^{2}}\left(-\frac{3 e n}{1-e^{2}} \frac{\partial}{\partial n}+\sin \tau \frac{\partial}{\partial e}-\cos \tau \frac{1}{e} \frac{\partial}{\partial \theta}\right) \\
F_{2}(l, x)= & \frac{P^{2}}{W^{2}}\left(-\frac{3 W n}{1-e^{2}} \frac{\partial}{\partial n}+\left(\cos \tau+\frac{e+\cos \tau}{W}\right) \frac{\partial}{\partial e}\right. \\
& \left.+\left(\sin \tau+\frac{\sin \tau}{W}\right) \frac{1}{e} \frac{\partial}{\partial \theta}\right)
\end{aligned}
$$

with $W=1+e \cos \tau$. The pulsation is

$$
\omega(l, x)=\frac{n W^{2}}{\left(1-e^{2}\right)^{3 / 2}} .
$$

Averaging on the base space eliminates $l$, that is the drift in the equation.

The normal maximized Hamiltonian on $\mathbf{S}^{1} \times T^{*} X$ is

$$
H(l, x, p)=\frac{\omega}{2}\left(H_{1}^{2}+H_{2}^{2}\right)(l, x, p),
$$

where $H_{i}=\left\langle p, F_{i}(l, x)\right\rangle, i=1,2$, are the Hamiltonian lifts of the vector fields. Let

$$
\bar{H}(x, p)=\frac{1}{2 \pi} \int_{0}^{2 \pi} H(l, x, p) \mathrm{d} l
$$

be the averaged Hamiltonian. As $1 / l_{f}$, the new small parameter, tends to zero, the flow of $\bar{H}$ converges uniformly towards the flow of $H$ on $\left[0, l_{f}\right]$. (See [15].) It turns out that the averaged flow is Riemannian. We refer to $[5,9]$ for details.

Proposition 2. The averaged Hamiltonian is

$$
H(x, p)=\frac{p_{r}^{2}}{2}+\frac{c^{2}}{2 r^{2}}\left(\frac{1-\lambda \sin ^{2} \varphi}{\sin ^{2} \varphi} p_{\theta}^{2}+p_{\varphi}^{2}\right)
$$

with $r=(2 / 5) n^{5 / 6}, c=\sqrt{2 / 5}$ and $\lambda=4 / 5$. 
The metric is

$$
\mathrm{d} r^{2}+\frac{r^{2}}{c^{2}}\left(\frac{\sin ^{2} \varphi}{1-\lambda \sin ^{2} \varphi} \mathrm{d} \theta^{2}+\mathrm{d} \varphi^{2}\right)
$$

It is Liouville integrable. The integration and the analysis of optimality can be made on the restriction to $\mathbf{S}^{2}$ by reparameterizing time according to $\mathrm{d} s=$ $c^{2} \mathrm{~d} l / r^{2}$. (See [6].) This amounts to restricting to a coarser moduli space where homothetic ellipses are identified. The restricted metric is

$$
X R(\lambda X) \mathrm{d} \theta^{2}+\mathrm{d} \varphi^{2} \quad \text { with } \quad R=\frac{1}{1-X} \quad \text { and } \quad X=\sin ^{2} \varphi
$$

As $\chi\left(\mathbf{S}^{2}\right)=2$, the two vector fields $(X R(X))^{-1 / 2} \partial / \partial \theta, \partial / \partial \varphi$ cannot form a global frame on the sphere. They have polar singularities that do not define genuine singularities of the metric.

Remark 2. Coordinates $(\theta, \varphi)$ are associated with the covering of the sphere with two punctures at North and South poles,

$$
\mathbf{R} \times(0, \pi) \ni(\theta, \varphi) \mapsto(\sin \varphi \cos \theta, \sin \varphi \sin \theta, \cos \varphi) \in \mathbf{R}^{3}
$$

One retrieves the standard covering $\exp : \mathbf{C} \rightarrow \mathbf{C}^{*} \simeq \mathbf{S}^{2}-\{N, S\}$ by putting $(\theta, \varphi) \mapsto \tan (\varphi / 2) \exp (i \theta)$.

The Hamiltonian on $\mathbf{S}^{2}$ is $H_{2}=(1 / 2)\left[(X R(\lambda X))^{-1} p_{\theta}^{2}+p_{\varphi}^{2}\right]$. On the level $H_{2}=1 / 2$, time is arc length and we get the quadrature

$$
Y^{2}=4(1-X)\left[X-p_{\theta}^{2}(1-\lambda X)\right], \quad Y=\dot{X}
$$

Since $\theta$ is cyclic, $p_{\theta}$ is constant (Clairaut first integral of a surface of revolution). The complex curve is of genus zero and admits a rational parameterization. We get

$$
\sin z=\frac{1}{\delta^{2}-p_{\theta}^{2}}\left[2 \delta^{2} X-\left(\delta^{2}+p_{\theta}^{2}\right)\right], \quad \mathrm{d} t=\frac{\mathrm{d} z}{2 \delta},
$$

for $z \in \mathbf{R}$ and $\delta^{2}=1+\lambda p_{\theta}^{2}$. We set $\theta_{0}=0$ by symmetry of revolution. We also assume $\varphi_{0}=\pi / 2$ without loss of generality since time translations generate any extremal on $H_{2}=1 / 2$ with arbitrary initial condition. The squared adjoint $p_{\theta}^{2}$ is bounded by $1 /(1-\lambda)$.

Proposition 3. The transcendence for two bodies and two controls is harmonic. One has

$$
\begin{gathered}
\sin ^{2} \varphi=\frac{1}{2 \delta^{2}}\left[\left(\delta^{2}-p_{\theta}^{2}\right) \cos (2 \delta t)+\left(\delta^{2}+p_{\theta}^{2}\right)\right], \quad \delta^{2}=1+\lambda p_{\theta}^{2}, \quad \lambda=4 / 5 \\
\theta=\operatorname{sign}\left(p_{\theta}\right)\left[\operatorname{atan} \frac{\left(\delta^{2}-p_{\theta}^{2}\right)+\left(\delta^{2}+p_{\theta}^{2}\right) \tan (\delta t+\pi / 4)}{2 \delta p_{\theta}}\right]_{0}^{t}-\lambda p_{\theta} t .
\end{gathered}
$$


Proof. The quadrature on $\theta$ is

$$
\frac{\mathrm{d} \theta}{\mathrm{d} z}=\frac{p_{\theta}}{2 \delta}\left(\frac{1}{X}-\lambda\right)
$$

whence the result.

Coordinate $\varphi$ (resp. $\theta)$ is periodic (resp. quasi-periodic) with period $T=$ $2 \pi / \delta=2 \pi / \sqrt{1+\lambda p_{\theta}^{2}}$. (The period of $\varphi$ is twice the period of $X=\sin ^{2} \phi$.) The increment of $\theta$ over one period is important for the optimality analysis concluding the section. One has

$$
\Delta \theta=2 \pi\left(1-\frac{\lambda p_{\theta}}{\sqrt{1+\lambda p_{\theta}^{2}}}\right) .
$$

Fix $y_{0}$ on $\mathbf{S}^{2}$. The exponential mapping is defined for $t \in \mathbf{R}$ and $p_{0} \in$ $H_{2}\left(y_{0}, \cdot\right)^{-1}(1 / 2) \subset T_{y_{0}}^{*} \mathbf{S}^{2}$ by

$$
\exp _{y_{0}}:\left(t, p_{0}\right) \mapsto \Pi \circ \exp t \vec{H}_{2}\left(y_{0}, p_{0}\right)=y\left(t, y_{0}, p_{0}\right)
$$

where $\Pi: T^{*} \mathbf{S}^{2} \rightarrow \mathbf{S}^{2}$ is the canonical projection and $\vec{H}_{2}$ the symplectic gradient. A conjugate point is a critical value of the exponential mapping. The time associated with the critical point is the conjugate time, and one can define the first conjugate point along the geodesic associated with a given $p_{0}$. The (first) conjugate locus is the set of all such points on geodesics emanating from $y_{0}$. The standard Jacobi theorem [13] asserts that, up to the first conjugate point, a geodesic is locally minimizing with respect to neighbouring continuous broken curves with same endpoints.

Theorem 2. In the two-body two-control case, the conjugate locus of any point on the sphere has four (possibly degenerate) cusps, two horizontal and two meridional.

Proof. According to [10] result, a sufficient condition is that $\Delta \theta$ is strictly decreasing convex. The condition is valid for (8).

Finally, define the cut time along the geodesic defined by $p_{0}$ as the supremum of times $t$ such that the geodesic $s \mapsto \exp _{y_{0}}\left(s, p_{0}\right)$ is globally minimizing on $[0, t]$. The corresponding point, if any, is the cut point. The cut locus is the set of all such points on geodesics emanating from $y_{0}$. It is known since Poincaré that the cut locus of an analytic metric on the sphere is a finite tree whose extremities are singularities of the conjugate locus. In the case of a metric with more symmetries, the result can be specialized as follows.

Theorem 3 ([24]). The cut locus of an analytic metric on the sphere of revolution with equatorial symmetry is an antipodal subarc provided the Gauss curvature is nondecreasing from the North pole to the equator. 


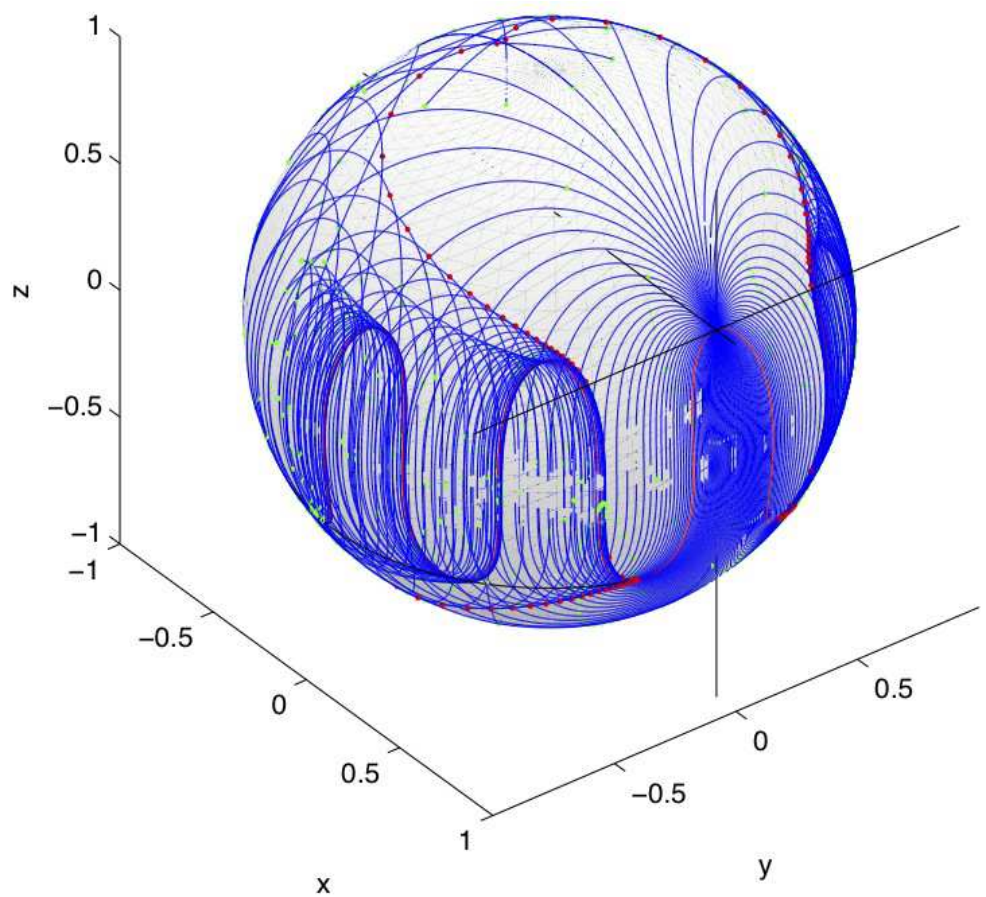

Fig. 1. Conjugate locus, two bodies and two controls. The astroid-shaped locus (in red) is the envelope of geodesics (in blue) emanating from the initial point. It has four (degenerate for initial condition on the poles) cusps, two horizontal and two meridional. The cut locus is a closed antipodal subarc (in black) whose extremities are horizontal cusps of the conjugate locus.

Though metric (7) has the required symmetries, the monotonicity condition on the curvature does not hold as

$$
K=\frac{1-\lambda(3-2 X)}{(1-\lambda X)^{2}}
$$

is not decreasing when $X \in[0,1]$ (remember that $X=\sin ^{2} \varphi$ ) for $\lambda=4 / 5$. A refined result relying on $\Delta \theta$ being strictly decreasing still gives the result [10].

Theorem 4. In the two-body two-control case, the cut locus of any point on the sphere is a closed antipodal subarc.

Varying $\lambda$ from zero to one in the definition of the metric (7), one connects the canonical metric on the sphere to a metric with an equatorial singularity,

$$
\frac{\sin ^{2} \varphi}{1-\sin ^{2} \varphi} \mathrm{d} \theta^{2}+\mathrm{d} \varphi^{2}
$$


The original metric is conformal to the standard metric on an oblate ellipsoid of revolution with semi-minor axis $\sqrt{1-\lambda}$ since

$$
\left.X R(\lambda X) \mathrm{d} \theta^{2}+\mathrm{d} \varphi^{2}=\frac{1}{1-\lambda \sin ^{2} \varphi}\left[\mathrm{d} \theta^{2}+(1-\lambda) \sin ^{2} \varphi\right) \mathrm{d} \varphi^{2}\right] .
$$

Making $\lambda$ tend to one can be interpretated as letting the semi-minor axis tend to zero, thus collapsing the sphere on a two-face disk [7]. Such a singularity is intrinsic in the case of only one control as explained in next section.

\section{Two bodies, one control}

Consider the $\mathrm{L}^{2}$-minimization of the two-body problem with only one control acting tangentially [8],

$$
\ddot{q}+\frac{q}{|q|^{3}}=u \frac{\dot{q}}{|\dot{q}|}, \quad u \in \mathbf{R}, \quad \int_{0}^{t_{f}}|u|^{2} \mathrm{~d} t \rightarrow \min .
$$

The state space is as before the trivial fiber space $\mathbf{S}^{1} \times X, X=\mathbf{R}_{+}^{*} \times \mathbf{S}^{2}$, but we correct the relation between $\varphi$ and the eccentricity,

$$
e=\sin \varphi \sqrt{1+\cos ^{2} \varphi}
$$

Changing again time to cumulated longitude,

$$
\frac{\mathrm{d} x}{\mathrm{~d} l}=u F_{1}(l, x), \quad \int_{0}^{l_{f}}|u|^{2} \frac{\mathrm{d} l}{\omega(l, x)} \rightarrow \min \quad\left(\text { fixed } l_{f}\right) .
$$

In $(n, e, \theta)$ coordinates,

$$
F_{1}=-\frac{3\left(1-e^{2}\right) w}{n^{1 / 3}(1+e \cos \tau)^{2}} \frac{\partial}{\partial n}+\frac{2\left(1-e^{2}\right)^{2}}{n^{4 / 3}(1+e \cos \tau)^{2} w}\left[(e+\cos \tau) \frac{\partial}{\partial e}+\frac{\sin \tau}{e} \frac{\partial}{\partial \theta}\right]
$$

with true anomaly $\tau=l-\theta$ and $w=\sqrt{1+2 e \cos \tau+e^{2}}$. Since the drift is unchanged, the pulsation is the same (compare (5)),

$$
\omega(l, x)=\frac{n(1+e \cos \tau)^{2}}{\left(1-e^{2}\right)^{3 / 2}} .
$$

The normal maximized Hamiltonian on $\mathbf{S}^{1} \times T^{*} X$ is

$$
H(l, x, p)=\frac{\omega}{2} H_{1}^{2}(l, x, p),
$$

where $H_{1}=\left\langle p, F_{1}(l, x)\right\rangle$. Define the averaged Hamiltonian as before (6). It is remarkable that the averaged flow remains Riemannian. 
Proposition 4. The averaged Hamiltonian is

$$
H(x, p)=\frac{p_{r}^{2}}{2}+\frac{c^{2}}{2 r^{2}}\left[\frac{\left(1-\sin ^{2} \varphi\right)^{2}}{\sin ^{2} \varphi\left(2-\sin ^{2} \varphi\right)^{2}} p_{\theta}^{2}+p_{\varphi}^{2}\right]
$$

with $r=(2 / 5) n^{5 / 6}$ and $c=2 / 5$.

As in the case of two controls, the flow is Liouville integrable and the whole analysis can be restricted to $\mathbf{S}^{2}$. The metric induced on the sphere is

$$
X R(X) \mathrm{d} \theta^{2}+\mathrm{d} \varphi^{2}, \quad R(X)=\frac{1}{4}\left[1+\frac{2}{1-X}+\frac{1}{(1-X)^{2}}\right], \quad X=\sin ^{2} \varphi .
$$

There is now an equatorial singularity at $\varphi=\pi / 2$. It is an order two pole at $X=1$ of the rational fraction $R$. (Compare with $R=1 /(1-X)$ in the previous section.)

Let $H_{2}=(1 / 2)\left[(X R(X))^{-1} p_{\theta}^{2}+p_{\varphi}^{2}\right]$. On the level $H_{2}=1 / 2$, the quadrature on $\varphi$ is

$$
Y^{2}=4(1-X)\left[X(2-X)^{2}-4 p_{\theta}^{2}(1-X)^{2}\right], \quad Y=\dot{X} .
$$

The underlying curve is of genus one. ${ }^{3}$ It is parameterized by a doubly periodic Weierstraß function,

$$
X=1-\frac{1}{\wp(z)-1 / 3}, \quad \frac{\mathrm{d} t}{\mathrm{~d} z}=1+\frac{1}{\wp(z)-1 / 3},
$$

whose invariants reflect the dependence on $p_{\theta}$,

$$
g_{2}=\frac{16}{3}+16 p_{\theta}^{2}, \quad g_{3}=\frac{64}{27}-\frac{16}{3} p_{\theta}^{2} .
$$

Without loss of generality, we restrict again the computation to $\theta_{0}=0$ and $\varphi_{0}=\pi / 2$. With the initial condition at singularity, $p_{\theta}^{2}$ is unbounded in contrast to the two-control case. Analyzing roots of the degree three polynomial $4 \xi^{3}-g_{2} \xi-g_{3}$ associated with Weierstraß function, one sees that the parameterization has to be restricted to the unbounded component of the cubic to ensure $X \in[0,1]$. Hence $z$ belongs to $\mathbf{R}$.

Proposition 5. The transcendence for two bodies and one (tangential) control is elliptic. One has

$$
\begin{gathered}
\sin ^{2} \varphi=\frac{\wp(z)-4 / 3}{\wp(z)-1 / 3}, \quad z \in \mathbf{R}, \\
t=\frac{1}{\wp^{\prime}(a)}\left[\ln \frac{\sigma(z-a)}{\sigma(z+a)}\right]_{0}^{z}+\left(1+\frac{2 \zeta(a)}{\wp^{\prime}(a)}\right) z, \\
\theta=2 p_{\theta}\left[\frac{1}{\wp^{\prime}(b)} \ln \frac{\sigma(z-b)}{\sigma(z+b)}-\frac{1}{\wp^{\prime}(c)} \ln \frac{\sigma(z-c)}{\sigma(z+c)}\right]_{0}^{z}+4 p_{\theta}\left(\frac{\zeta(b)}{\wp^{\prime}(b)}-\frac{\zeta(c)}{\wp^{\prime}(c)}\right) z, \\
\text { with } \wp(a)=1 / 3, \wp(b)=4 / 3, \wp(c)=-2 / 3, \text { and invariants (12). }
\end{gathered}
$$

${ }^{3}$ Excluding the degenerate case $p_{\theta}=0$ associated with meridians. 
Proof. The quadrature on $\theta$ is

$$
\frac{\mathrm{d} \theta}{\mathrm{d} z}=2 p_{\theta}\left(\frac{1}{\wp(z)-4 / 3}-\frac{1}{\wp(z)+2 / 3}\right) .
$$

It is similar to the quadrature (11) on $t$. Introducing Weierstraß $\zeta$ and $\sigma$ functions, $\wp=-\zeta^{\prime}, \zeta=\sigma^{\prime} / \sigma$, one has

$$
\int \frac{\wp^{\prime}(a) \mathrm{d} z}{\wp(z)-\wp(a)}=2 \zeta(a) z+\ln \frac{\sigma(z-a)}{\sigma(z+a)},
$$

whence the result.

The family of genus one complex curves (10) are all homeomorphic to the torus. The topological classification of extremals is then trivial. We recall standard facts on the moduli space of elliptic curves [17] so as to refine the classification up to conformal equivalence.

Let $L$ be a lattice in the complex plane with base $\left(l_{1}, l_{2}\right)$ (complex numbers linearly independent over $\left.\mathbf{R}^{2}\right)$. A pair $\left(l_{1}^{\prime}, l_{2}^{\prime}\right)$ defines another base if only if

$$
\begin{aligned}
& l_{1}^{\prime}=a l_{1}+b l_{2}, \\
& l_{2}^{\prime}=c l_{1}+d l_{2},
\end{aligned}
$$

for some matrix

$$
\left[\begin{array}{ll}
a & b \\
c & d
\end{array}\right] \in \mathrm{SL}(2, \mathbf{Z})
$$

Two tori $\mathbf{C} / L, \mathbf{C} / L^{\prime}$ are conformal if and only if there is some $\mu \in \mathbf{C}^{*}$ such that $L^{\prime}=\mu L$. Let $\left(l_{1}, l_{2}\right)$ and $\left(l_{1}^{\prime}, l_{2}^{\prime}\right)$ be bases of $L$ and $L^{\prime}$, respectively. We can assume that $\tau=l_{2} / l_{1}$ and $\tau^{\prime}=l_{2}^{\prime} / l_{1}^{\prime}$ belong to Poincaré upper half-plane, H. From the previous remarks, $L$ and $L^{\prime}$ are conformal if and only if there is a quadruple $(a, b, c, d)$ such that

$$
\tau^{\prime}=\frac{a \tau+b}{c \tau+d}, \quad a, b, c, d \in \mathbf{Z}, \quad a d-b c=1 .
$$

Such particular Möbius transformations are automorphisms of $\mathbf{H}$. The induced group morphism between $\mathrm{SL}(2, \mathbf{Z})$ and $\operatorname{Aut}(\mathbf{H})$ has kernel \pm id. Transformations (13) are then identified with the Fuchsian modular group $\operatorname{PSL}(2, \mathbf{Z})=$ $\mathrm{SL}(2, \mathbf{Z}) / \pm \mathrm{id}$. Then $\mathbf{H} / \operatorname{PSL}(2, \mathbf{Z})$ is the moduli space for congruences of conformal tori. One eventually defines the modular function

$$
j(\tau)=\frac{g_{2}^{3}}{\Delta}
$$

It is a bijection from $\mathbf{H} / \operatorname{PSL}(2, \mathbf{Z})$ onto $\mathbf{C}$.

In our case, to each $p_{\theta}$ is associated a real rectangular lattice. Using (12), one can define

$$
j\left(p_{\theta}\right)=\frac{16\left(1+3 p_{\theta}^{2}\right)^{3}}{27 p_{\theta}^{2}\left(8+13 p_{\theta}^{2}+16 p_{\theta}^{4}\right)}
$$

and obtain the following classification of extremals. 
Proposition 6. There are not more than three conformal $\varphi$-curves.

Proof. Function (14) has exactly two critical points, so $j\left(p_{\theta}\right)=$ constant has at most three distinct solutions (not taking into account symmetric solutions, $\left.\pm p_{\theta}\right)$.

To estimate the conjugate locus at singularity, we use the following local model. Set $x=\pi / 2-\varphi, y=\theta$. The metric (9) is locally approximated by

$$
\mathrm{d} x^{2}+\frac{\mathrm{d} y^{2}}{x^{2 p}}
$$

In the case of one (tangential) control, $p=2$.

Proposition 7 ([7]). The conjugate locus at the origin of (15) is $y=$ $\pm C_{p} x^{p+1}$ minus the origin itself. As $p \rightarrow \infty, C_{p} \sim 8 /(3 p+1)$.

As a result, the conjugate locus of the metric on $\mathbf{S}^{2}$ has an order 3 contact with the equatorial singularity. Because of the symmetry $p_{\theta} \rightarrow-p_{\theta}$, this defines two meridional cusps of the conjugate locus at $\varphi_{0}=\pi / 2$. (See Fig. 2.) The result of section 2 can be extended to this singular setting.

Theorem 5 ([7]). If $\Delta \theta$ is strictly decreasing convex, the conjugate locus has four (possibly degenerate) cusps, all meridional for equatorial points, two horizontal and two meridional otherwise.

The verification on $\Delta \theta$ is intricate but can be made numerically. The condition holds asymptotically when $p_{\theta}$ tends to 0 or $\infty$. The following estimates are computed in [7],

$$
\Delta \theta \sim_{0} 2 \pi\left(1-\frac{3 \sqrt{2}}{4} p_{\theta}+\frac{35 \sqrt{2}}{128} p_{\theta}^{3}\right), \quad \Delta \theta \sim_{\infty} \frac{4}{3}(2-\sqrt{2}) K(3-2 \sqrt{2}) p_{\theta}^{-3 / 2},
$$

where $K(k)$ is the complete Jacobi integral of first kind and modulus $k$.

Theorem $6([7])$. If $\Delta \theta$ is strictly decreasing, the cut locus of a point on the sphere is the equator minus the point itself for equatorial points, a closed antipodal subarc otherwise.

\section{Three bodies, two controls}

In contrast with sections 2 and 3, we keep the original constraint on the control, and consider the final time minimization of

$$
\ddot{q}+\nabla V_{\mu}(q)+2 i q=\varepsilon u, \quad|u| \leq 1
$$

See [9] for preliminary computations on the $\mathrm{L}^{2}$-relaxation of the problem. Available results on controlled three-body problems are mostly numerical. 


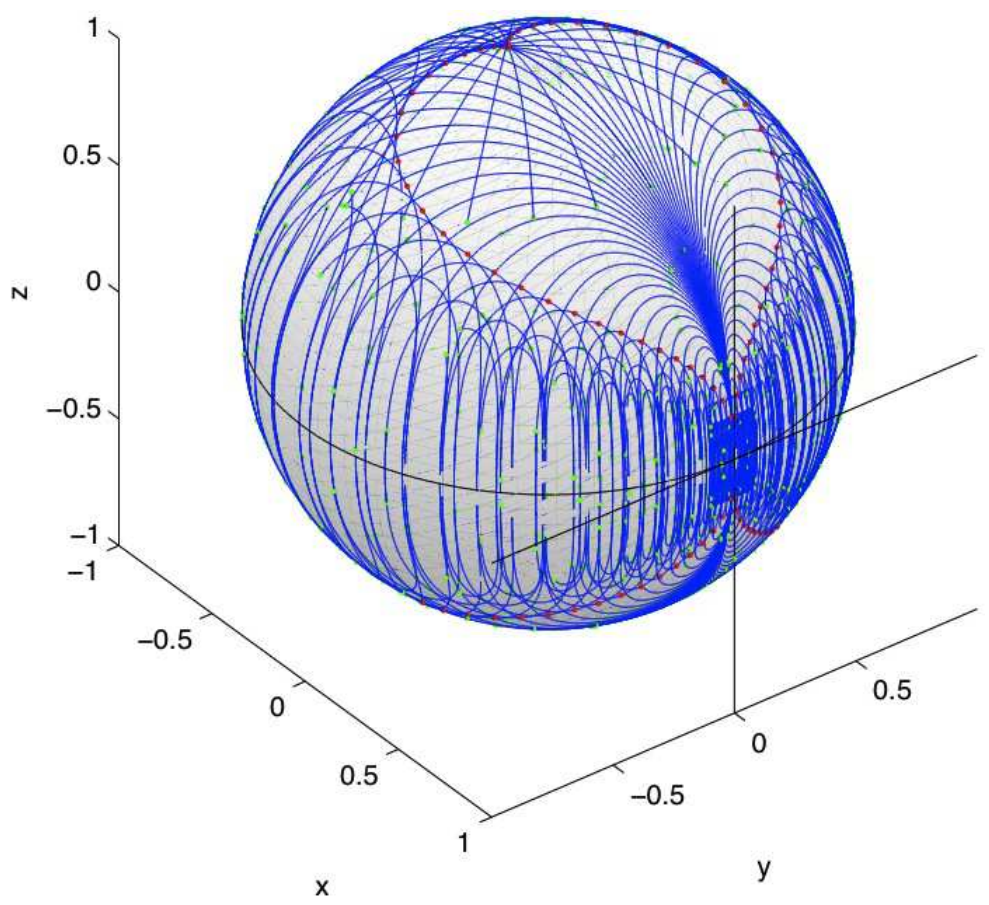

Fig. 2. Conjugate locus, two bodies and one (tangential) control. The double-heart locus (in red) is the envelope of geodesics (in blue) emanating from the initial point. It has four meridional cusps (two of them generated by order 3 contacts at origin). The cut locus (in black) is the whole equator minus the origin.

They usually deal with refined models taking into account three-dimensional effects, perturbations, and rely on direct optimization methods. (See, e.g., [3].)

The position vector $q$ belongs to the complex plane with two punctures at $-\mu$ and $1-\mu$, denoted $Q_{\mu}$. The state space $X_{\mu}$ is the tangent space $T Q_{\mu}$ in (rotating) cartesian coordinates $(q, \dot{q})$. It is the cotangent $T^{*} Q_{\mu}$ in $(q, p)$ variables, see (1). In both cases, $X_{\mu} \simeq Q \times \mathbf{R}^{2}$. In cartesian coordinates,

$$
\dot{x}=F_{0}(x)+\varepsilon\left(u_{1} F_{1}(x)+u_{2} F_{2}(x)\right), \quad|u| \leq 1 .
$$

There,

$$
F_{0}(x)=\dot{q} \frac{\partial}{\partial q}-\left(\nabla V_{\mu}(q)+2 i q\right) \frac{\partial}{\partial \dot{q}}, \quad F_{1}(x)=\frac{\partial}{\partial \dot{q}_{1}}, \quad F_{2}(x)=\frac{\partial}{\partial \dot{q}_{2}} .
$$

The maximized normal Hamiltonian ${ }^{4}$ is

$$
H=-1+H_{0}+\varepsilon \sqrt{H_{1}^{2}+H_{2}^{2}}, \quad H_{i}=\left\langle p, F_{i}(x)\right\rangle, \quad i=0, \ldots, 2 .
$$

\footnotetext{
${ }^{4}$ From now on, $p$ denotes the adjoint to $x$.
} 
Extremals are classified according to the order of their contact with the switching surface $\Sigma=\left\{H_{1}=H_{2}=0\right\}$.

Proposition 8. Contacts with $\Sigma$ are of order one and define isolated $\pi$ singularities. ${ }^{5}$

Proof. The distribution $\left\{F_{1}, F_{2}\right\}$ being involutive, the switching function $\psi=$ $\left(H_{1}, H_{2}\right)$ is $\mathscr{C}^{1}$,

$$
\dot{\psi}_{1}=\left\{H_{0}, H_{1}\right\}-u_{1}\left\{H_{1}, H_{2}\right\}, \quad \dot{\psi}_{2}=\left\{H_{0}, H_{2}\right\}+u_{2}\left\{H_{1}, H_{2}\right\} .
$$

The bracket $\left\{H_{1}, H_{2}\right\}$ vanishes on $\Sigma$. The drift comes from a second order mechanical system, so $\left\{F_{1}, F_{2},\left[F_{0}, F_{1}\right],\left[F_{0}, F_{2}\right]\right\}$ has full rank. Then $\dot{\psi} \neq 0$ on $\Sigma$ and contacts are of order one. By Pontryagin maximization condition, $u=\psi /|\psi|$, so $u$ is changed to $-u$ whenever $\psi$ vanishes.

As $\Sigma$ is of codimension two in $T^{*} X_{\mu}$, we neglect $\pi$-singularities and restrict the computation to smooth extremals not crossing the switching surface.

For the minimum time problem, the exponential mapping associated with order zero extremals is defined on a neighbourhood of the origin in $\mathbf{R} \times$ $H\left(x_{0}, \cdot\right)^{-1}(0)$,

$$
\exp _{x_{0}}:\left(t, p_{0}\right) \mapsto \Pi \circ \exp t \vec{H}\left(x_{0}, p_{0}\right)=x\left(t, x_{0}, p_{0}\right), \quad \Pi: T^{*} X_{\mu} \rightarrow X_{\mu} .
$$

Given a target $x_{f}$, the problem is to find a zero of the shooting equation

$$
\exp _{x_{0}}\left(t_{f}, p_{0}\right)=x_{f} .
$$

Two-body problems are embedded in three-body ones thanks to parameter $\mu$. This paves the way for using continuation methods between two and threebody control problems. (See also [14] for such an approach in the two-body case.) Rather than information on the adjoint, the knowledge of the Kepler minimum time from [12] turns out to be critical to initialize the continuation. Our target for numerical computation is an equilibrium point of the uncontrolled problem or Lagrange point [25]. Such points where the influences of the two primaries compensate each other are appropriate targets for the continuation. Here we use the $L_{2}$ Lagrange point. It is equal to the second primary when $\mu=0$. Lagrange points are extensively studied in celestial mechanics and mission design [18]. A second continuation on $\varepsilon$ is also used to reach low bounds on the control, see results Fig. 3. Hill regions are projections on the $q$-space of level sets of the Jacobi integral. In the controlled case, they vary dynamically along the trajectory (see Fig. 4),

$$
R_{\mu}(t)=\left\{\xi \in Q_{\mu} \mid J_{\mu}(q(t), \dot{q}(t))-V_{\mu}(\xi) \geq 0\right\} .
$$

A normal extremal is regular if it verifies the strong Legendre condition, $\partial^{2} H / \partial u^{2}<0$ on $\left[0, t_{f}\right]$.

\footnotetext{
${ }^{5}$ Instantaneous rotations of angle $\pi$ of the control [12].
} 

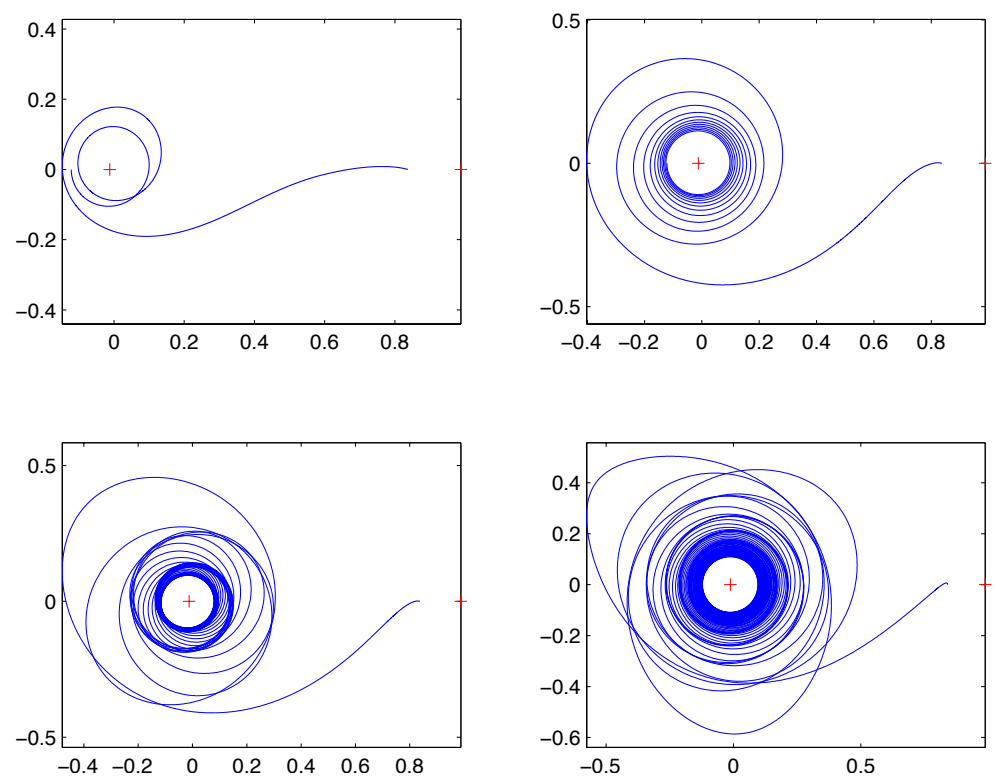

Fig. 3. Three bodies, two controls. Minimum time trajectories from the geostationary orbit to Lagrange $L_{2}$ point in the Earth-Moon system $(\mu \simeq 1.21 e-2)$. Successively, $\varepsilon=2.440 e-0,2.440 e-1,1.220 e-1$ and $4.148 e-2$.

Lemma 1. Order zero extremals of the minimum time three-body problem with two controls are regular.

Proof. Along an order zero extremal, $u \in \mathbf{S}^{1}$. In any chart,

$$
\frac{\partial^{2} H}{\partial u^{2}}=-\varepsilon \sqrt{H_{1}^{2}+H_{2}^{2}}=-\varepsilon|\psi| .
$$

The absence of $\pi$-singularity implies the strengthened Legendre condition.

As in the Riemannian case (without singularities), regular extremals are locally time minimizing for short times [1]. To investigate further local optimality, one generalizes Jacobi theory to the optimal control setting. Define again conjugate points as critical values of the exponential mapping. The following technical condition is sufficient to avoid degenerate situations on the kernel of the second variation of the problem (see [23]). Let

$$
E_{x_{0}}:\left(t_{f}, u\right) \mapsto x\left(t_{f}, x_{0}, u\right)
$$

be the endpoint mapping. It is defined on a neighbourhood of the reference pair $\left(t_{f}, u\right)$ in $\mathbf{R} \times \mathrm{L}^{\infty}\left(\left[0, t_{f}\right], \mathbf{S}^{1}\right)$. We assume that, for any subinterval $\left[t_{1}, t_{2}\right]$ of $\left[0, t_{f}\right]$, the partial derivative $\partial E_{x\left(t_{1}\right)} / \partial u\left(t_{2}-t_{1}, u \mid\left[t_{1}, t_{2}\right]\right)$ has corank one. 

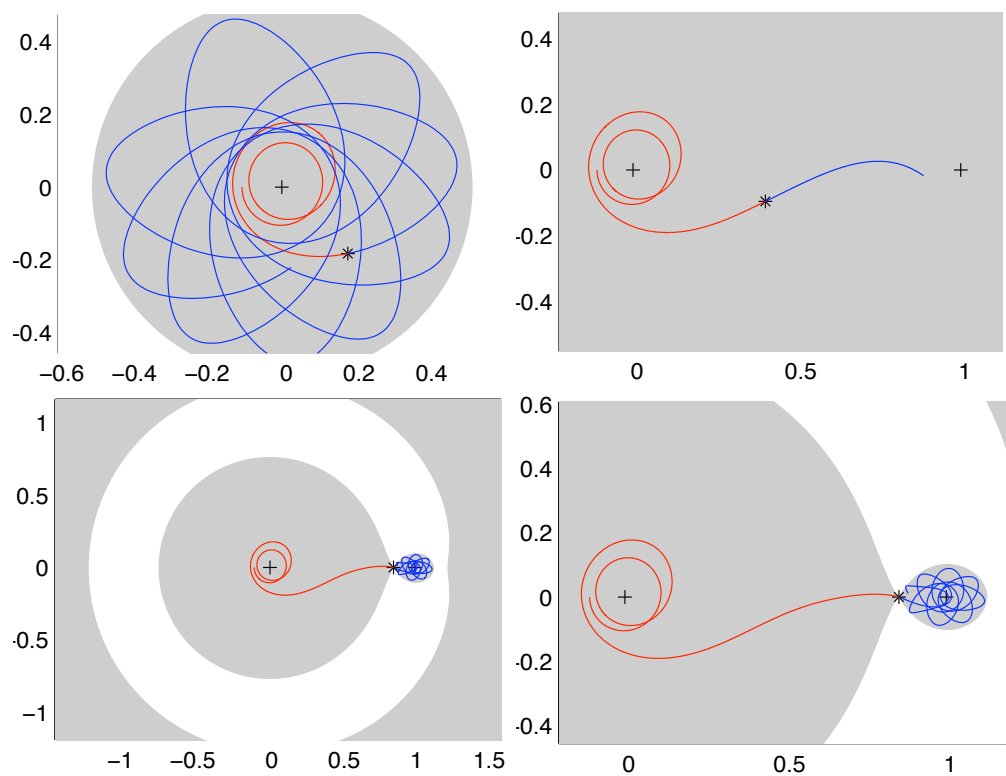

Fig. 4. Dynamics of the Hill regions, $\varepsilon=2.440 e-0$. The controlled trajectory (in red) is plotted up to three different times and prolongated by the osculating uncontrolled trajectory (in blue). During a first phase, energy $J_{\mu}$ is increased so as to include the $L_{2}$ target. The second phase is close to the motion of the system towards projection in the $q$-space of the Lagrange point. The last two graphs are identical (the rightmost one has a finer scale) and illustrate instability of the $L_{2}$ point after the target is reached.

Theorem $7([\mathbf{1}, \mathbf{2 3}])$. Under the corank one assumption, the trajectory associated with a regular extremal is $\mathscr{C}^{0}$-locally time minimizing up to the first conjugate point. Past this point, the control is not even $\mathrm{L}^{\infty}$-locally minimizing.

Local optimality of every extremal is verified by a conjugate point test. (See Fig. 5). The practical computation of conjugate points is done by rank evaluation on Jacobi fields [11]. The concept of conjugate point is extended by the notion of focal point [Ibid.] to encompass the case of submanifold targets. Such an example for a lunar orbit target is provided Fig. 6 .

Whatever the target, the value function $\varepsilon \mapsto t_{f}(\varepsilon)$ of the minimum time problem is decreasing: The smaller $\varepsilon$, the larger the transfer time. This is contradicted by results portrayed Fig. 7 . We infer that the first extremal is locally but not globally minimizing. When decreasing the bound on the control $\varepsilon$ from $2.221 e-1$ to $2.196 e-1$, one revolution around the first primary has to be added before escape towards the second body is obtained. There lies global analysis of the problem, in the interplay between the two small parameters $\mu$, $\varepsilon$. This leaves open the question of global optimality. 


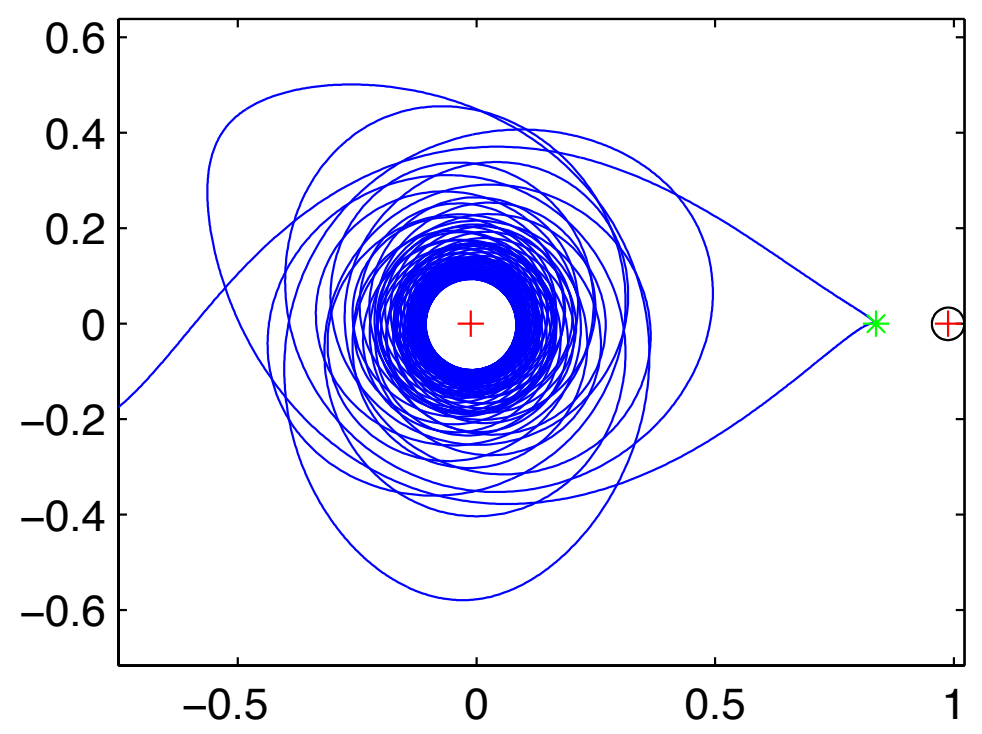

Fig. 5. Conjugate point computation, $\varepsilon=4.148 e-2$. The reference trajectory is prolongated up to the first conjugate point, beyond the $L_{2}$ target. Local optimality up to the target is guaranteed. The cuspidal point of first kind observed is generically due to the condition $\dot{q}_{f}=0$.
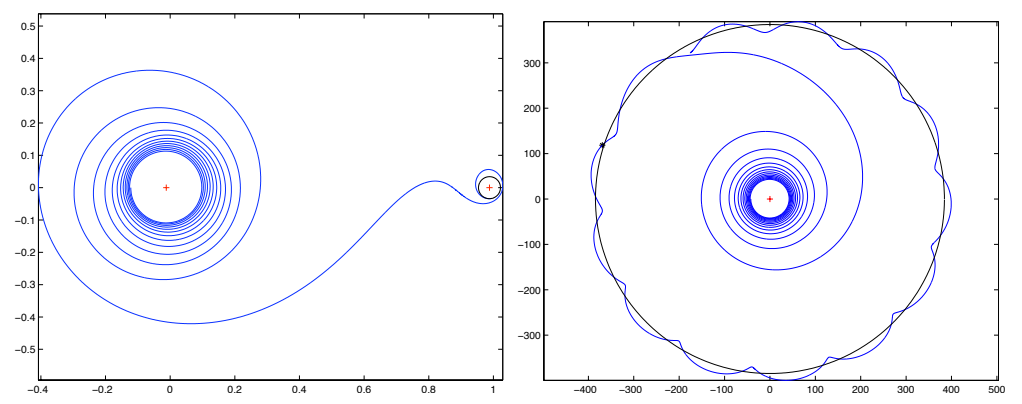

Fig. 6. Focal point computation, $\varepsilon=2.440 e-1$. The target is a lunar orbit, and the focal point test ensures local optimality of the trajectory. The leftmost frame is the rotating frame, the rightmost one is fixed.

\section{References}

1. Agrachev, A. A.; Sachkov, Y. L. Control Theory from the Geometric Viewpoint. Springer, 2004.

2. Belbruno, E. A. Two-body motion under the inverse square central force and equivalent geodesic flows. Celest. Mech. 15 (1977), no. 4, 467-476.

3. Betts, J. T.; Erb, S. O. Optimal Low Thrust Trajectories to the Moon. SIAM J. Appl. Dyn. Syst. 2 (2003), no. 2, 144-170. 

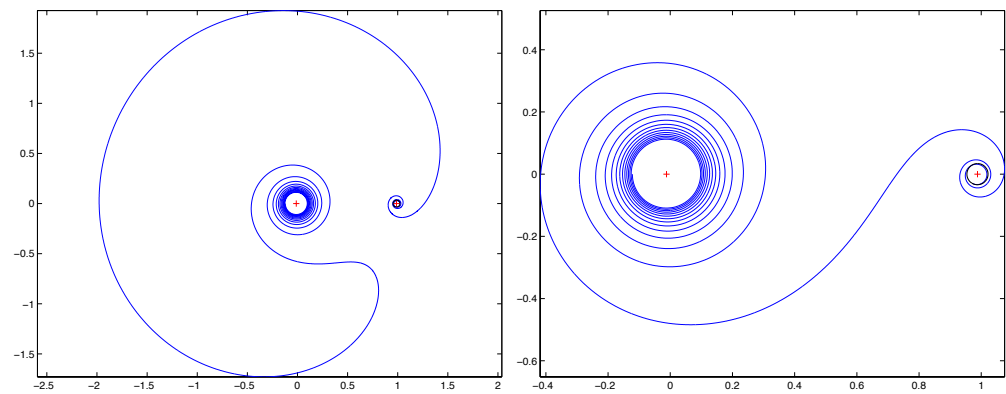

Fig. 7. Lunar target, $\varepsilon=2.221 e-1$ and $2.196 e-1$. Focal point tests ensure local optimality in both cases. However, $t_{f} \simeq 17.8$ versus $t_{f} \simeq 10.8$ in the second one. The first extremal is a local but not a global minimizer. The difference in strategies is apparent as one extra revolution around the Earth is added in the second case before reaching the lunar orbit target.

4. Bombrun, A.; Chetboun, J.; Pomet, J.-B. Transfert Terre-Lune en poussée faible par contrôle feedback. La mission SMART-1. INRIA Research report (2006), no. 5955, 1-27.

5. Bonnard, B.; Caillau, J.-B. Riemannian metric of the averaged energy minimization problem in orbital transfer with low thrust. Ann. Inst. H. Poincaré Anal. Non Linéaire 24 (2007), no. 3, 395-411.

6. Bonnard, B.; Caillau, J.-B. Geodesic flow of the averaged controlled Kepler equation. Forum math. 21 (2009), no. 5, 797-814.

7. Bonnard, B; Caillau, J.-B. Singular metrics on the two-sphere in space mechanics. HAL preprint (2008), no. 00319299, 1-25.

8. Bonnard, B.; Caillau, J.-B.; Dujol, R. Energy minimization of single-input orbit transfer by averaging and continuation. Bull. Sci. Math. 130 (2006), no. 8, 707719.

9. Bonnard, B.; Caillau, J.-B.; Picot, G. Geometric and numerical techniques in optimal control of the two and three-body problems. HAL preprint (2010), no. 00432631, 1-39.

10. Bonnard, B.; Caillau, J.-B.; Sinclair, R.; Tanaka, M. Conjugate and cut loci of a two-sphere of revolution with application to optimal control. Ann. Inst. H. Poincaré Anal. Non Linéaire 26 (2009), no. 4, 1081-1098.

11. Bonnard, B.; Caillau, J.-B.; Trélat, E. Second order optimality conditions in the smooth case and applications in optimal control. ESAIM Control Optim. and Calc. Var. 13 (2007), no. 2, 207-236. (apo.enseeiht.fr/cotcot.)

12. Caillau, J.-B.; Noailles, J. Coplanar control of a satellite around the Earth. ESAIM Control Optim. and Calc. Var. 6 (2001), 239-258.

13. Do Carmo, M. P. Riemannian geometry. Birkhuser, Boston, 1992.

14. Gergaud, J.; Haberkorn, T. Homotopy method for minimum consumption orbit transfer problem. ESAIM Control Optim. Calc. Var. 12 (2006), no. 2, 294-310.

15. Guckenheimer, J.; Holmes, P. Nonlinear oscillations, dynamical systems and bifurcations of vector fields. Springer, 1993.

16. Hatcher, A. Algebraic topology. Cambridge University Press, 2002.

17. Jones, G. A.; Singerman, D. Complex Functions. An Algebraic and Geometric Viewpoint. Cambridge University Press, 1987. 
18. Marsden, J. E.; Ross, S. D. New methods in celestial mechanics and mission design, Bull. Amer. Math. Soc. (N.S.) 43 (2006), no. 1, 43-73.

19. McCord, C.; Meyer, K. R.; Offin, D. Are Hamiltonian Flows Geodesic Flows? Trans. Amer. Math. Soc. 355 (2003), no. 3, 1237-1250.

20. Moser, J. K. Regularization of Kepler's problem and the averaging method on a manifold. Comm. Pure Appl. Math. 23 (1970), 609-635.

21. Osipov, Y. The Kepler problem and geodesic flows in spaces of constant curvature. Celestial Mech. 16 (1977), no. 2, 191-208.

22. Racca, G.; et al. SMART-1 mission description and development status. Planetary and space science 50 (2002), 1323-1337.

23. Sarychev, V. A. The index of second variation of a control system. Mat. Sb. 41 (1982), 338-401.

24. Sinclair, R.; Tanaka, M. The cut locus of a two-sphere of revolution and Toponogov's comparison theorem. Tohoku Math. J. 59 (2007), no. 2, 379-399.

25. Szebehely, V. Theory of orbits: The restricted problem of three bodies. Academic Press, 1967. 